\title{
Os determinantes psicológicos do consumidor verde
}

\author{
The psychological determinants of the green consumer
}

\section{Fernando Oliveira-Brochado}

Universidade de Lisboa (UL), Instituto Superior de Economia e Gestão (ISEG), Departamento de Gestão, Rua do Quelhas, n.ำ6, 1200-781 Lisboa, Portugal, fjbrochado@iseg.utl.pt

\section{Ana Oliveira-Brochado}

Instituto Universitário de Lisboa, ISCTE Business School, Business Research Unit (BRU-IUL), Departamento de Marketing, Operações e Gestão Geral, 1649-026 Lisboa, Portugal, ana.brochado@iscte.pt

\section{Tiago Caldeira}

Universidade Europeia, Laureate International Universities, 1500-210 Lisboa, Portugal, t.caldeira@hotmail.com

\section{Resumo}

Nos últimos anos tem-se verificado uma preocupação crescente em relação à temática ambiental na população em geral, nos consumidores, nos governos, nas empresas e nos investigadores. Efetivamente, os consumidores têm consciência de que o seu comportamento é suscetível de influenciar o meio ambiente, começando a ter uma maior preocupação aquando da escolha dos produtos que adquirem e consomem. Os governos procuram promover políticas de sustentabilidade ambiental, e as empresas reconhecem que a adoção de um posicionamento socialmente responsável e a oferta de produtos verdes suscetíveis de responder às necessidades do consumidor são aspetos chave para o desenvolvimento e manutenção de uma vantagem competitiva. Neste contexto, o presente trabalho teve como objetivo estudar os determinantes do comportamento de compra de produtos verdes, com base no quadro teórico da Teoria do Comportamento Planeado. Considerando uma amostra de colaboradores de uma empresa em Portugal com cobertura geográfica nacional, concluiu-se que as variáveis atitude, norma subjetiva e controlo percebido têm influência na intenção comportamental, de compra de produtos verdes.

Palavras-chave: Consumidor verde, equações estruturais, Portugal, produtos verdes, teoria do comportamento planeado.

\begin{abstract}
During the last years, environmental issues have received increasing attention by the general population, consumers, governments, companies and researchers. Effectively, consumers are now aware that their behavior is likely to influence the environment, and thus have a greater concern when they choose products to buy and consume. Governments attempt to promote policies of environmental sustainability, and companies recognize that the adoption of a socially responsible positioning and the offering of green products are critical factors to develop and maintain a competitive advantage. In this context, the present work intends to study the determinants of purchase behavior of green products based on the theoretical framework of the Theory of Planned Behaviour. Based on a sample of employees of a Portuguese company in Portugal with national geographic coverage, we concluded that the variables attitude, subjective norm, and perceived control influence the behavioral intention of buying green products.
\end{abstract}

Keywords: Green consumer, green products, Portugal, structural equation modeling, theory of planned behavior.

\section{Introdução}

Nos últimos anos tem-se verificado uma preocupação crescente em relação à temática ambiental entre a população em geral, os consumidores, os governos, as empresas e os investigadores. Assim, a população tem reconhecido que o seu comportamento é suscetível de influenciar o meio ambiente e os consumidores tornaram-se mais preocupados com os seus hábitos diários e com as repercussões que estes podem ter no meio ambiente, começando a ter uma maior preocupação aquando da escolha dos produtos que adquirem e consomem. Os governos procuram promover políticas de sustentabilidade ambiental, e as empresas reconhecem que a adoção de um posicionamento socialmente responsável (Carroll, 1991) e a oferta de produtos verdes suscetíveis de responder às necessidades do consumidor (Gupta \& Ogden, 2009) são aspetos chave para o desenvolvimento e manutenção de uma vantagem competitiva (Wahid, Rahbar, \& Shyan, 2011), mesmo num cenário de crise económica (Miras-Rodríguez, Escobar-Perez \& Gallego, 2015). Este facto tem-se refletido no aumento do consumo de produtos verdes, e tem impulsionado os marketers a aprofundar o conceito de marketing verde (Pujari \& Wright, 1996).

Na literatura, tem surgido um número crescente de estudos sobre o comportamento do consumidor verde, quer em termos de descrição do seu perfil demográfico, socioeconómico e psicográfico, quer em termos de modelização do comportamento e da intenção de aquisição de produtos verdes (e.g., no caso português, os trabalhos de Paço \& Raposo, $2008 \mathrm{e}$ 2009; Akehurst, Afonso, \& Gonçalves, 2012; Marques \& Almeida, 2013; Gonçalves, Menezes \& Marques, 2015). No entanto, alguns estudos (e.g., Kalafatis, Pollard, East \& Tsogas, 1999; Litvine \& Wüstenhagen, 2011) concluíram que a presença de uma atitude pro-ambientalista não conduz, necessariamente, a comportamentos amigos do ambiente (i.e., comportamentos verdes).

Deste modo, revela-se importante aprofundar a discussão e a compreensão dos determinantes da intenção e do comportamento do consumidor verde. Dos vários modelos que procuram explicar comportamentos pro-ambientais, um dos mais conhecidos é a Teoria do Comportamento Planeado 
(TCP), desenvolvida por Ajzen $(1985,1991)$. A TCP pode ser usada para compreender e prever o comportamento de aquisição de produtos verdes e para apoiar o desenvolvimento de programas no sentido de promover atividades pro-ambientais (Litvine \& Wüstenhagen, 2011). Efetivamente, para além da atitude pro-ambiental, relacionada com a aquisição de produtos verdes, a TCP assume outros determinantes da intenção de comportamento verde, como sejam, o controlo percebido e a norma subjetiva.

Assim, o presente trabalho tem como objetivo estudar os determinantes da intenção e do comportamento de compra de produtos verdes (e.g., atitudes, normas sociais e controlo percebido) com base na TCP.

De seguida, é apresentada uma síntese da TCP e a listagem de alguns trabalhos que a usaram no estudo do comportamento do consumidor verde. Depois são descritas as opções metodológicas, nomeadamente em termos de hipóteses, população-alvo, macro e micro estrutura do questionário desenvolvido, e métodos estatísticos usados. Segue-se a análise e discussão dos resultados e finaliza-se com uma conclusão.

\section{Teoria do comportamento planeado}

Quando se fala em comportamento verde do consumidor, existe uma fonte importante que deve ser considerada, a Teoria do Comportamento Planeado (Ajzen, 1985, 1991). Esta teoria surgiu no âmbito da psicologia social e tem sido utilizada por diversos autores para explicar o comportamento e as decisões de compra dos consumidores, fornecendo resultados robustos (Han \& Hansen, 2012) através de modelos conceptualmente simples (Litvine \& Wüstenhagen, 2011).

A Teoria do Comportamento Planeado (TCP) (Ajzen, 1985, 1991) é derivada da Teoria da Acção Racional (TAR) (Ajzen \& Fishbein, 1980), sendo ambas uma extensão do modelo multiatributos de Fishbein and Ajzen (1975). A TCP baseiase na premissa de que os indivíduos fazem uso racional da informação disponível quando tomam decisões comportamentais (Conner \& Armitage, 1998).

A Teoria do Comportamento Planeado prevê nas suas aplicações as crenças comportamentais, as crenças normativas e as crenças de controlo. Tal como a TAR, a TCP considera as intenções comportamentais como mediadoras da relação atitude-comportamento. Ou seja, o comportamento é manifestado pelas intenções pessoais em executá-lo. A TCP, no entanto, é considerada uma extensão à TAR ao incluir o controlo percebido do comportamento, como determinante das intenções. Esta teoria baseou-se na premissa de que mantendo uma intenção constante, um maior controlo percebido irá aumentar a probabilidade de que se realize o comportamento de compra com sucesso, quando surgem as oportunidades. Ou seja, existindo um determinado grau de controlo do comportamento dos indivíduos, estes tendem a realizar as suas intenções quando as oportunidades aparecem. 0 controlo real sobre o comportamento, refere-se à capacidade que os indivíduos possuem em realizar realmente um determinado comportamento.

Assim, na TCP o comportamento é determinado pelas intenções e as intenções comportamentais são determinadas por três fatores (Figura 1): (i) atitude em relação ao comportamento - a avaliação favorável ou desfavorável do consumidor atribuída à realização do comportamento; (ii) norma subjetiva - a perceção individual da pressão social de realizar ou não um comportamento, i.e., as expetativas dos grupos sociais (familiares, amigos, colegas); e (iii) controlo percebido - a avaliação individual da capacidade de realização do comportamento. 0 controlo percebido também pode influenciar diretamente o comportamento.

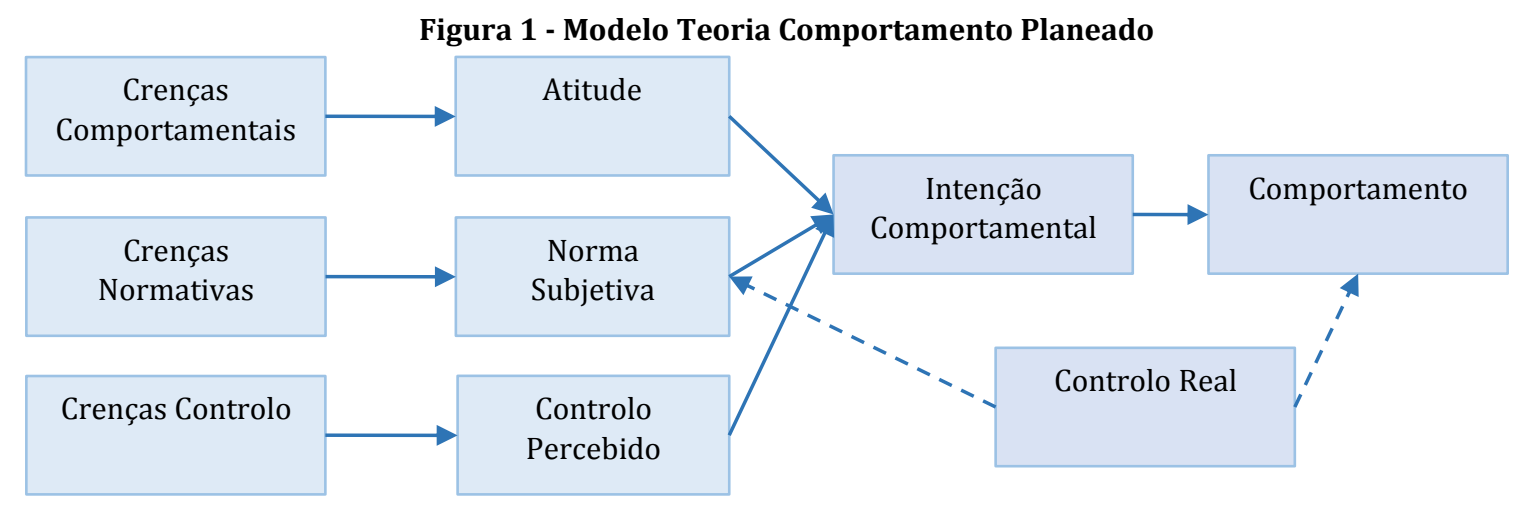

Fonte: Adaptado a partir de Ajzen $(1985,1991)$

A TCP foi utilizada para explicar comportamentos proambientais, como a reciclagem e a separação de lixo doméstico (e.g., Cheung, Chan \& Wong, 1999; Biswas, Licata, McKee, Pullig \& Daughtridge, 2000; Chu \& Chui, 2003; Kaiser \& Gutscher, 2003; Oreg \& Katz-Gerro, 2006; Davis, O'Callaghan \& Knox, 2009; Botetzagias, Dima \& Malesios, 2015), o consumo de energias renováveis (Litvine \& Wüstenhagen, 2011), a utilização de transportes públicos (Bamberg \& Schmidt, 1998; Heath \&
Gifford, 2002), a redução do uso de veículos privados por questões ambientais (Oreg \& Katz-Gerro, 2006), o consumo de alimentos orgânicos (Sparks \& Shepherd, 1992; Han \& Hansen, 2012), a escolha de hotéis ecológicos (Han \& Kim, 2010; Han, Hsu \& Sheu 2010; Kim \& Han, 2010; Chen \& Tung, 2014) e a compra de produtos verdes (Kalafatis et al., 1999; Chan \& Lau, 2002; Davis et al., 2009; Moser, 2015). 


\section{Metodologia}

\subsection{Hipóteses de investigação}

A relação entre o comportamento do consumidor verde e a intenção de compra, e entre esta e a atitude, a norma subjetiva e o controle percebido serão efetuadas considerando o modelo definido a partir da Teoria do Comportamento Planeado (TCP) desenvolvido por Ajzen (1985). Neste contexto, foram definidas as seguintes hipóteses de investigação:

H1. Há uma relação positiva entre a atitude em relação à compra verde e a intenção de compra verde;

H2. Há uma relação positiva entre a norma subjetiva e a intenção de compra verde;

H3. Há uma relação positiva entre o controle percebido da compra verde e a intenção de compra verde;

H4. Há uma relação positiva entre a intenção de compra verde e o comportamento de compra verde.

\subsection{Desenvolvimento do questionário}

O questionário desenvolvido inicia-se com a apresentação do seguinte conceito de produto verde ao consumidor: "Considere os produtos verdes como aqueles que são fabricados segundo uma política deliberada de gestão ambiental, onde a produção é efetuada segundo critérios de eficiência energética, conservação de recursos naturais, preocupação com a saúde do consumidor, segurança ambiental, facilidade de reutilização, durabilidade $e$ biodegradabilidade. - Considere produtos verdes que se encontram disponíveis no mercado português".

0 questionário encontra-se estruturado, em termos de macroestrutura, em três secções. Na primeira, uma questão geral e introdutória solicita a avaliação dos hábitos de compra de produtos verdes por parte dos consumidores. A segunda secção inclui questões relativas às variáveis extraídas do Modelo do Comportamento Planeado (i.e., a atitude em relação à compra de produtos verdes, a norma subjetiva, o controlo percebido do comportamento, a intenção de comportamento e o comportamento de compra verde). Todos os itens das variáveis latentes que integram o modelo foram medidos através de uma escala Likert de sete pontos, sendo a respetiva fonte apresentada na Figura 2 que se segue. Para as variáveis atitude, norma subjetiva e comportamento o valor mínimo (1) corresponde à categoria 'discordo totalmente' e o valor máximo (7) a 'concordo totalmente'. As categorias mínima (1) e máxima (7) da variável intenção de compra são usadas para designar 'nunca' e 'sempre', respetivamente. Relativamente ao comportamento de compra, o valor mínimo e máximo representam, respectivamente, 'nunca' e 'sempre' para a frequência de compra, 'nada' e 'muito' para os gastos em produtos verdes e ' 0 ' e 'mais de 10' para o número de produtos verdes adquiridos.

Figura 2 - Construtos utilizados na construção do questionário

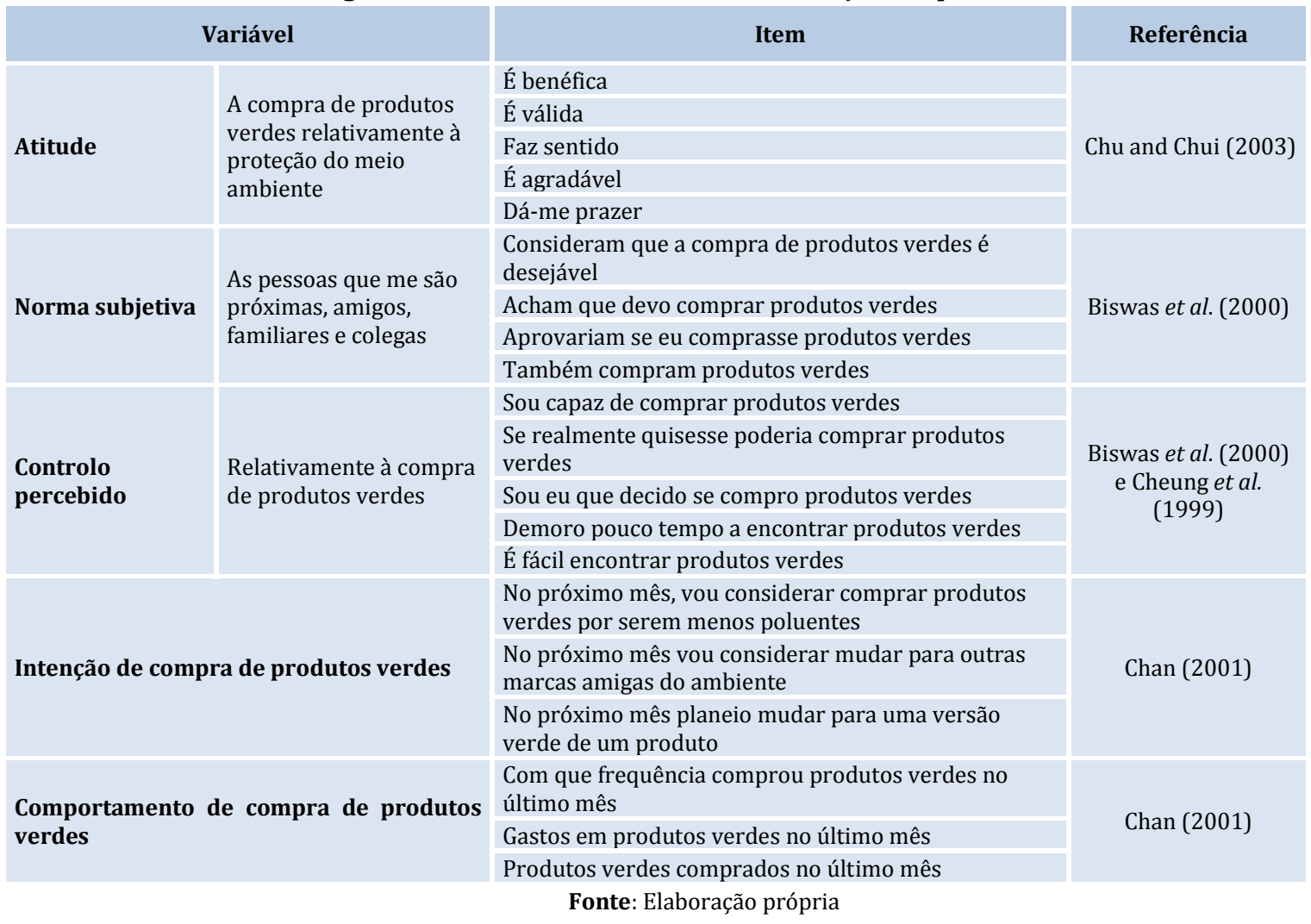

Por fim, são colocadas questões relativas ao perfil do indivíduo, em termos de variáveis demográficas (sexo, idade e estado civil) e sócio-económicas (habilitações e rendimento).

\subsection{Processo de amostragem e métodos estatísticos}

No presente estudo a população alvo é constituída por consumidores portugueses que colaboram numa empresa 
portuguesa de implementação nacional, de ambos os sexos, com idade superior a 18 anos e residentes em Portugal.

A recolha de dados foi efetuada através de um questionário divulgado numa versão on-line, via e-mail, para todos os colaboradores da empresa, sendo obtidas 282 respostas válidas (taxa de resposta de 28\%) através de um procedimento de amostragem, não aleatório, por conveniência (Malhotra, 2010).

Na caracterização estatística do perfil dos inquiridos foram utilizadas tabelas de frequência e estatísticas descritivas. A abordagem de equações estruturais foi utilizado no sentido de testar as relações entre as variáveis (Hair, Tatham, Anderson \& Black, 2009).

\section{Análise e discussão dos resultados}

\subsection{Perfil demográfico e sócio-económico dos respondentes}

No presente estudo foi utilizada uma amostra composta por um conjunto diversificado de indivíduos com mais de 18 anos, a residir em Portugal, com o fator comum de trabalharem na mesma empresa. Após a aplicação do questionário, foi possível obter uma amostra, caracterizada em termos demográficos e sócio-económicos no quadro que se segue (Quadro 1).

A amostra é constituída por 282 respondentes, em que 167 são do sexo feminino $(59,2 \%)$ e 115 respondentes do sexo masculino (40,8\%). A idade média é de 44,1 (D.P.=10,4), variando entre 18 e 70 anos. No que respeita às habilitações, observa-se que cerca de $74 \%$ dos indivíduos possuem pelo menos o grau de licenciatura. Relativamente à variável rendimento verificou-se que 171 dos respondentes $(60,6 \%)$ aufere entre $€ 1.001$, a $€ 2.000$, seguido de 65 inquiridos $(23,0 \%)$ que aufere entre $€ 2.001$ $\mathrm{a} € 4.000$. Os restantes inquiridos auferem entre $€ 500$ a $€$ $1.000(10,3 \%)$ e mais de $€ 4.000(6,0 \%)$. Em relação ao estado civil podemos observar que 184 respondentes são casados $(65,2 \%)$, seguidos de 66 inquiridos $(23,4 \%)$ que são solteiros, 28 respondentes (9,9\%) são divorciados, sendo que 4 dos inquiridos são viúvos.

Quadro 1 - Perfil demográfico e socioeconómico dos inquiridos $(\mathrm{N}=282)$

\begin{tabular}{|c|c|c|c|c|c|c|}
\hline \multirow{2}{*}{ Variável } & \multirow{2}{*}{ Categorias } & \multicolumn{2}{|c|}{ Frequência } & \multirow{2}{*}{ Moda } & \multirow{2}{*}{ Média } & \multirow{2}{*}{ Desvio-padrão } \\
\hline & & Absoluta & Relativa (\%) & & & \\
\hline \multirow{2}{*}{ Sexo } & Masculino & 115 & 40,8 & & & \\
\hline & Feminino & 167 & 59,2 & $\mathrm{X}$ & & \\
\hline Idade & & & & & 44,1 & 10,4 \\
\hline \multirow{5}{*}{ Habilitações } & $3^{\circ}$ Ciclo & 5 & 1,8 & & & \\
\hline & Ensino secundário & 68 & 24,1 & & & \\
\hline & Licenciatura & 177 & 62,8 & $\mathrm{X}$ & & \\
\hline & Mestrado & 30 & 10,6 & & & \\
\hline & Doutoramento & 2 & 0,7 & & & \\
\hline \multirow{4}{*}{ Rendimento mensal } & $€ 500-€ 1.000$ & 29 & 10,3 & & & \\
\hline & $€ 1.001-€ 2.000$ & 171 & 60,6 & $\mathrm{X}$ & & \\
\hline & $€ 2.001-€ 4.000$ & 65 & 23,0 & & & \\
\hline & $>€ 4.000$ & 17 & 6,0 & & & \\
\hline \multirow{4}{*}{ Estado civil } & Casado & 184 & 65,2 & $\mathrm{X}$ & & \\
\hline & Solteiro & 6 & 23,4 & & & \\
\hline & Divorciado & 28 & 9,9 & & & \\
\hline & Viúvo & 4 & 2,4 & & & \\
\hline
\end{tabular}

Fonte: Elaboração própria

\subsection{Comportamento verde e os seus determinantes}

Dos produtos verdes listados, os que registaram uma maior frequência de aquisição nos últimos três meses pelos inquiridos foram as lâmpadas economizadoras e os produtos biodegradáveis. Por outro lado, os produtos menos adquiridos são aqueles que têm um período de vida superior, como o carro amigo do ambiente ou o telemóvel ecológico (Quadro 2).

Quadro 2 - Frequência de compra de produtos verdes nos últimos 3 meses

\begin{tabular}{|c|c|c|c|c|c|c|c|c|c|c|c|c|}
\hline \multirow[t]{2}{*}{ Produto Verde } & \multicolumn{2}{|c|}{ Nunca (1) } & \multicolumn{2}{|c|}{$\begin{array}{c}\text { Poucas Vezes } \\
\text { (2) }\end{array}$} & \multicolumn{2}{|c|}{$\begin{array}{l}\text { Algumas } \\
\text { Vezes (3) }\end{array}$} & \multicolumn{2}{|c|}{$\begin{array}{c}\text { Muitas Vezes } \\
\text { (4) }\end{array}$} & \multicolumn{2}{|c|}{ Sempre (5) } & \multirow[t]{2}{*}{ Moda } & \multirow[t]{2}{*}{ Mediana } \\
\hline & Freq. & $\%$ & Freq. & $\%$ & Freq. & $\%$ & Freq. & $\%$ & Freq. & $\%$ & & \\
\hline Papel Reciclado & 59 & $21 \%$ & 59 & $21 \%$ & 109 & $39 \%$ & 45 & $16 \%$ & 10 & $4 \%$ & 3 & 3 \\
\hline Produtos em Embalagens & 13 & $5 \%$ & 46 & $16 \%$ & 106 & $38 \%$ & 102 & $36 \%$ & 15 & $5 \%$ & 3 & 3 \\
\hline Lâmpadas Economizadoras & 8 & $3 \%$ & 31 & $11 \%$ & 60 & $21 \%$ & 63 & $22 \%$ & 120 & $43 \%$ & 5 & 4 \\
\hline Eletrodomésticos Classe A+++ & 73 & $26 \%$ & 29 & $10 \%$ & 52 & $18 \%$ & 63 & $22 \%$ & 65 & $23 \%$ & 1 & 3 \\
\hline Roupa feita com material & 133 & $47 \%$ & 84 & $30 \%$ & 55 & $20 \%$ & 8 & $3 \%$ & 2 & $1 \%$ & 1 & 2 \\
\hline Telemóvel ecológico & 223 & $79 \%$ & 37 & $13 \%$ & 14 & $5 \%$ & 5 & $2 \%$ & 3 & $1 \%$ & 1 & 1 \\
\hline Carro amigo do Ambiente & 207 & $73 \%$ & 38 & $13 \%$ & 13 & $5 \%$ & 11 & $4 \%$ & 13 & $5 \%$ & 1 & 1 \\
\hline Material de escritório ecológico & 85 & $30 \%$ & 89 & $32 \%$ & 75 & $27 \%$ & 26 & $9 \%$ & 7 & $2 \%$ & 2 & 2 \\
\hline Produtos Biodegradáveis & 14 & $5 \%$ & 44 & $16 \%$ & 102 & $36 \%$ & 98 & $35 \%$ & 24 & $9 \%$ & 3 & 3 \\
\hline
\end{tabular}

Fonte: Elaboração própria 
Relativamente à intenção de comportamento e ao comportamento verde conseguimos depreender através do Quadro 3 que os inquiridos de uma forma geral têm intenção e comportamento de compra verde. Efetivamente, a maioria dos inquiridos identifica como benéfica a compra de produtos verdes relativamente ao meio ambiente. É referido que as pessoas mais próximas dos inquiridos aprovariam a compra de produtos verdes, considerando aqui a norma social, e a sua aceitação social. Relativamente à compra de produtos verdes, os inquiridos são capazes de tomar a decisão de comprar produtos verdes, ou seja, têm um controlo percebido relativamente à compra de produtos verdes. As variáveis atitude, norma subjetiva e controlo percebido, intenção de comportamento e comportamento de compra, foram medidos numa escala de Likert de 7 pontos. As pontuações médias dos itens variaram entre 3,32 e 6,34. Em termos relativos os itens que integram os determinantes da intenção de comportamento assumem valores médios superiores à intenção e esta relativamente ao comportamento.

Quadro 3 - Determinantes de intenção de comportamento e de comportamento verde

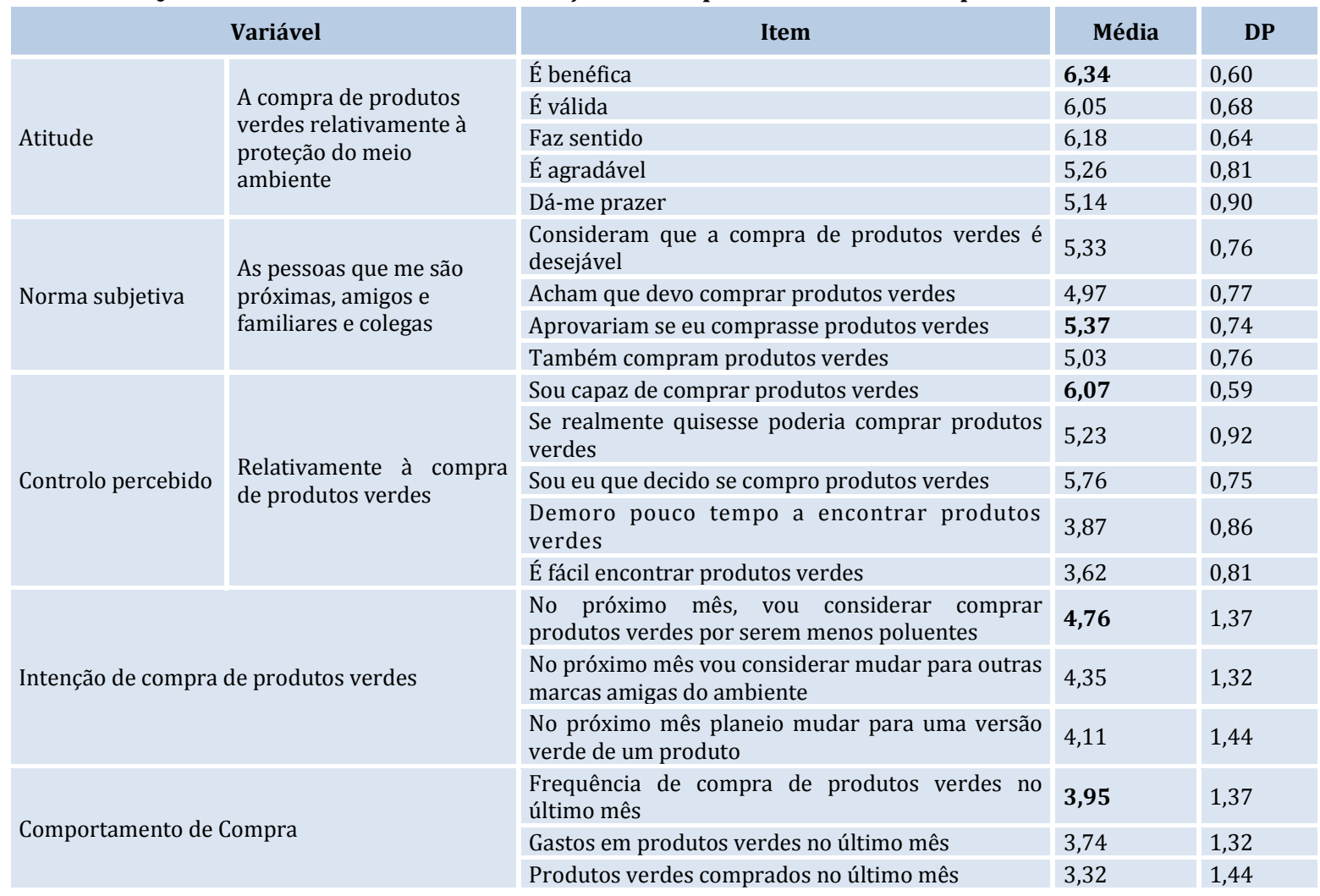

Fonte: Elaboração própria

\subsubsection{Análise fatorial e fiabilidade dos construtos}

De forma a selecionar os itens finais a utilizar na estimação do modelo confirmatório utilizaram-se duas técnicas: a primeira, a análise fatorial exploratória usando o método das componentes principais com rotação varimax, que é realizada a todos os itens dos construtos no sentido de se verificar, para a realidade em causa, se faz sentido manter a escala sem nenhum tipo de ajustamento e para verificar se existe correlação entre as variáveis confirmando a sua aplicação. A segunda, a análise do coeficiente Alpha de Cronbach, que avalia a fiabilidade na vertente consistência interna dos itens. Os valores da estatística Kaiser-MeyerOlkin (KMO) e os resultados do Teste de Bartlett para cada construto revelaram a adequação da análise fatorial (Quadro 4).

Quadro 4 - KMO e Teste de Bartlett

\begin{tabular}{|l|c|c|}
\hline & KMO & Teste de Bartlett*** \\
\hline Atitude & 0,73 & 631,00 \\
\hline Norma subjectiva & 0,82 & 557,42 \\
\hline Controlo percebido & 0,76 & 516,11 \\
\hline Intenção de compra de produtos verdes & 0,75 & 558,61 \\
\hline Comportamento de Compra & 0,69 & 336,93 \\
\hline
\end{tabular}

*** estatisticamente significativo a $1 \%$.

Fonte: Elaboração própria

Adicionalmente, são apresentados os pesos fatoriais obtidos com as análises fatoriais exploratórias realizadas e os coeficientes Alpha de Cronbach que relevaram fiabilidade adequada dos itens selecionados para integrar o modelo em estudo (Quadro 5). 
Quadro 5 - Análise fatorial exploratória e coeficiente alfa de Cronbach

\begin{tabular}{|c|c|c|c|c|c|c|c|}
\hline \multirow{2}{*}{\multicolumn{2}{|c|}{ Variável }} & \multirow{2}{*}{ Item } & \multicolumn{2}{|c|}{ Peso Fatorial } & \multirow{2}{*}{$\begin{array}{l}\text { Variância } \\
\text { explicada }\end{array}$} & \multirow{2}{*}{$\begin{array}{l}\text { Valor } \\
\text { próprio }\end{array}$} & \multirow{2}{*}{$\begin{array}{l}\text { Alpha de } \\
\text { Cronbach }\end{array}$} \\
\hline & & & F1 & F2 & & & \\
\hline \multirow{5}{*}{ Atitude } & \multirow{5}{*}{$\begin{array}{l}\text { A compra de } \\
\text { produtos verdes } \\
\text { relativamente à } \\
\text { proteção do } \\
\text { meio ambiente }\end{array}$} & É benéfica & 0,85 & & \multirow{5}{*}{$\begin{array}{l}58,18 \% \\
\text { (F1) e } \\
22,31 \% \\
\text { (F2) }\end{array}$} & \multirow{5}{*}{$\begin{array}{l}2,91(\mathrm{~F} 1) \mathrm{e} \\
1,16(\mathrm{~F} 2)\end{array}$} & \multirow{5}{*}{$0,80(\mathrm{~F} 1)$} \\
\hline & & É válida & 0,87 & & & & \\
\hline & & Faz sentido & 0,86 & & & & \\
\hline & & É agradável & & 0,88 & & & \\
\hline & & Dá-me prazer & & 0,91 & & & \\
\hline \multirow{4}{*}{$\begin{array}{l}\text { Norma } \\
\text { subjetiva }\end{array}$} & \multirow{4}{*}{$\begin{array}{l}\text { As pessoas que } \\
\text { me são } \\
\text { próximas, } \\
\text { amigos e } \\
\text { familiares e } \\
\text { colegas }\end{array}$} & $\begin{array}{l}\text { Consideram que a compra de produtos } \\
\text { verdes é desejável }\end{array}$ & 0,86 & & \multirow{4}{*}{$72,27 \%$} & \multirow{4}{*}{2,89} & \multirow{4}{*}{0,87} \\
\hline & & Acham que devo comprar produtos verdes & 0,90 & & & & \\
\hline & & $\begin{array}{l}\text { Aprovariam se eu comprasse produtos } \\
\text { verdes }\end{array}$ & 0,81 & & & & \\
\hline & & Também compram produtos verdes & 0,83 & & & & \\
\hline \multirow{5}{*}{$\begin{array}{l}\text { Controlo } \\
\text { percebido }\end{array}$} & \multirow{5}{*}{$\begin{array}{l}\text { Relativamente à } \\
\text { compra de } \\
\text { produtos verdes }\end{array}$} & Sou capaz de comprar produtos verdes & & 0,56 & \multirow{5}{*}{$\begin{array}{l}40,83 \% \\
(\mathrm{~F} 1) \mathrm{e} \\
27,45 \% \\
\quad(\mathrm{~F} 2)\end{array}$} & \multirow{5}{*}{$\begin{array}{l}2,04(\mathrm{~F} 1) \mathrm{e} \\
1,30(\mathrm{~F} 2)\end{array}$} & \multirow{5}{*}{$0,83(\mathrm{~F} 2)$} \\
\hline & & $\begin{array}{l}\text { Se realmente quisesse poderia comprar } \\
\text { produtos verdes }\end{array}$ & 0,60 & & & & \\
\hline & & $\begin{array}{l}\text { Sou eu que decido se compro produtos } \\
\text { verdes }\end{array}$ & 0,58 & & & & \\
\hline & & $\begin{array}{l}\text { Demoro pouco tempo a encontrar } \\
\text { produtos verdes }\end{array}$ & 0,75 & & & & \\
\hline & & É fácil encontrar produtos verdes & 0,72 & & & & \\
\hline \multirow{3}{*}{\multicolumn{2}{|c|}{$\begin{array}{l}\text { Intenção de compra de } \\
\text { produtos verdes }\end{array}$}} & $\begin{array}{l}\text { No próximo mês, vou considerar comprar } \\
\text { produtos verdes por serem menos } \\
\text { poluentes }\end{array}$ & 0,92 & & \multirow{3}{*}{84,52} & \multirow{3}{*}{2,54} & \multirow{3}{*}{0,91} \\
\hline & & $\begin{array}{l}\text { No próximo mês vou considerar mudar } \\
\text { para outras marcas amigas do ambiente }\end{array}$ & 0,93 & & & & \\
\hline & & $\begin{array}{l}\text { No próximo mês planeio mudar para uma } \\
\text { versão verde de um produto }\end{array}$ & 0,91 & & & & \\
\hline \multirow{3}{*}{\multicolumn{2}{|c|}{ Comportamento de Compra }} & $\begin{array}{l}\text { Frequência de compra de produtos verdes } \\
\text { nos últimos três meses }\end{array}$ & 0,90 & & \multirow{3}{*}{74,43} & \multirow{3}{*}{2,23} & \multirow{3}{*}{0,82} \\
\hline & & Gastos em produtos verdes no último mês & 0,89 & & & & \\
\hline & & $\begin{array}{l}\text { Produtos verdes comprados no último } \\
\text { mês }\end{array}$ & 0,80 & & & & \\
\hline
\end{tabular}

Fonte: Elaboração própria

\subsubsection{Análise fatorial confirmatória dos construtos}

O modelo revelou um bom ajustamento, sendo apresentados na tabela que se segue os pesos de cada item que integra a análise. A fiabilidade compósita (FC) dos factores revelou-se elevada, tendo variado entre 0,81 para o controlo percebido e 0,97 para a norma subjetiva. A variância extraída média (AVE), um indicador da validade convergente dos factores, revelou-se também adequada tendo variado entre 0,71 para o controlo percebido e a atitude e 0,78 para a norma subjetiva. A validade discriminante também se encontra assegurada, dado que para cada par de variáveis latentes os valores de AVE são superiores aos quadrados da correlação entre fatores (Quadro 6).

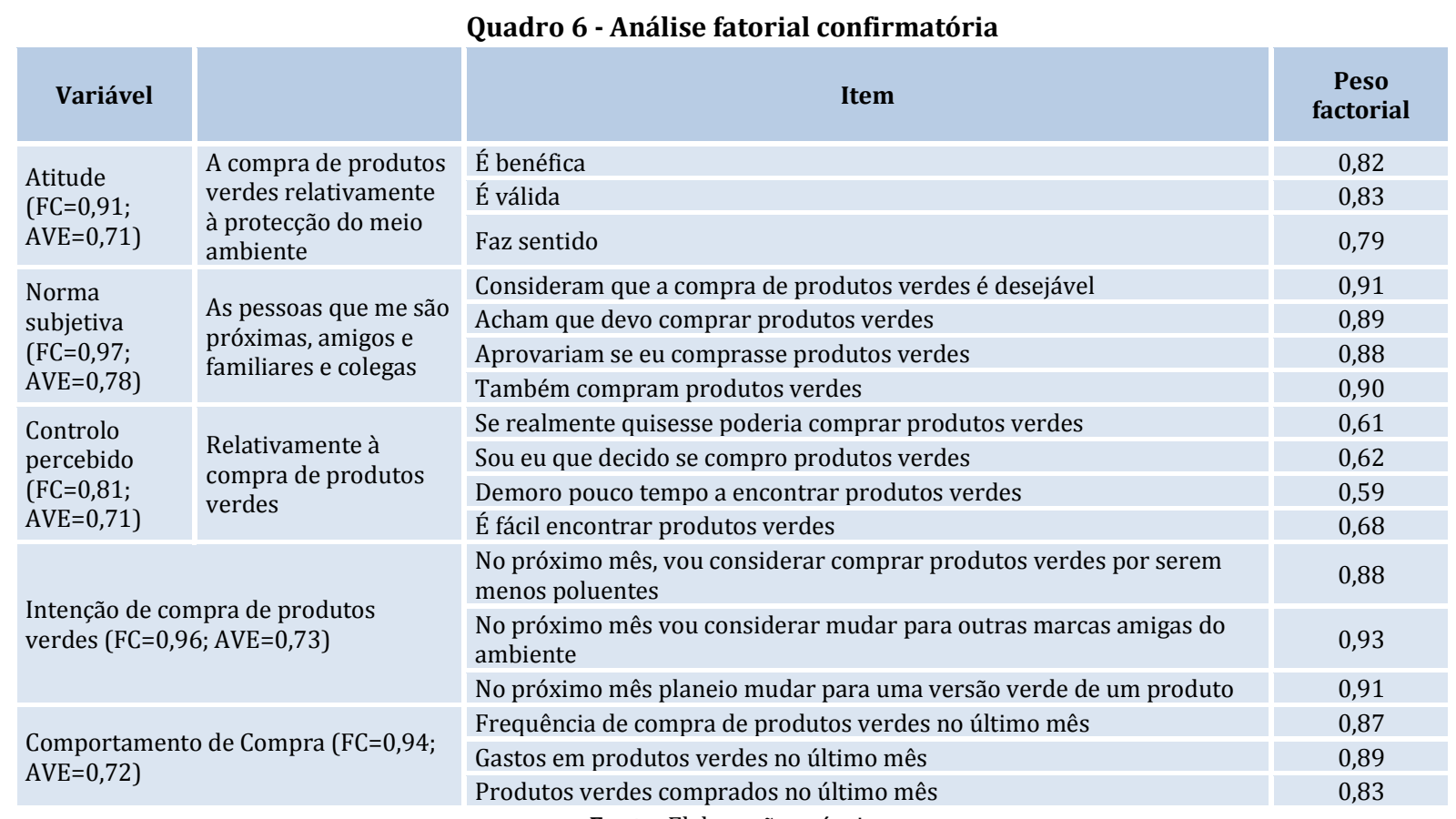

Fonte: Elaboração própria 


\subsubsection{Modelo de equações estruturais}

Após a análise da qualidade do ajustamento, foi estimado o modelo estrutural proposto, de modo a verificar se as hipóteses de pesquisa são confirmadas ou não. Após a estimação do modelo estrutural, é possível concluir que todas as hipóteses são suportadas.

As variáveis atitude, norma subjetiva e controlo percebido estabelecem uma relação positiva com a intenção de comportamento verde, ou seja, um incremento em cada uma destas variáveis irá aumentar a intenção e o comportamento verde. Por sua vez, a variável intenção de comportamento verde estabelece uma relação positiva com a variável comportamento verde, demonstrando assim que qualquer incremento numa das variáveis irá aumentar o comportamento de compra verde (Quadro 7).

\section{Quadro 7 - Modelo estrutural estimado}

\begin{tabular}{|l|c|c|c|}
\hline \multicolumn{1}{|c|}{ Hipóteses } & Estimativa & Valor $\boldsymbol{p}$ & Conclusão \\
\hline H1: Atitude -> Intenção de comportamento verde (+) & $0,49^{*}$ & 0,00 & Suportada \\
\hline H2: Norma subjetiva -> Intenção de comportamento verde (+) & $0,63^{*}$ & 0,00 & Suportada \\
\hline H3: Controlo percebido -> Intenção de comportamento verde (+) & $0,32^{* *}$ & 0,01 & Suportada \\
\hline H4: Intenção de comportamento verde -> Comportamento verde (+) & $0,40^{*}$ & 0,00 & Suportada \\
\hline Indicadores do ajustamento & & \\
\hline X $/$ gl & \multicolumn{2}{|c|}{3,96} & \\
\hline RMSEA & \multicolumn{2}{|c|}{0,069} & \\
\hline GFI & \multicolumn{2}{|c|}{0,91} & \\
\hline \% variância explicada do comportamento verde & \multicolumn{2}{|c|}{$5 \%$} & \\
\hline * Estatisticamente significativa a 1\%; ** estatisticamente significativa a 5\%. & & \\
\hline
\end{tabular}

Fonte: Elaboração própria

A Figura 3 esquematiza os determinantes do comportamento de compra de acordo com o modelo de equações estruturais.

Figura 3 - Determinantes do comportamento de compra de acordo com o modelo de equações estruturais

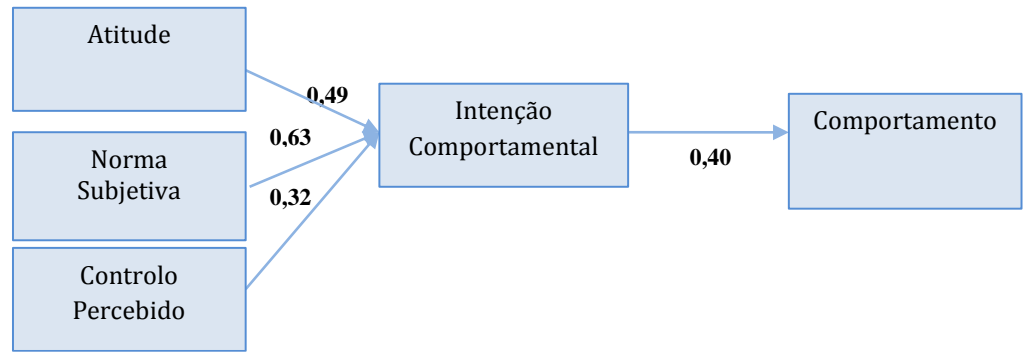

Fonte: Elaboração própria

\section{Conclusão}

No presente trabalho analisou-se a intenção e o comportamento de compra de produtos verdes usando como quadro teórico a Teoria do Comportamento Planeado. Com base numa amostra de 282 colaboradores de uma empresa pública portuguesa, concluiu-se que as quatro hipóteses de investigação foram suportadas. Assim, os resultados obtidos revelaram que as variáveis atitude, norma subjetiva e controlo percebido têm influência na intenção comportamental, que vão gerar um comportamento, que resulta na aquisição de produtos verdes. No entanto, apesar de no presente estudo se concluir que a atitude é uma variável relevante na explicação da intenção e do comportamento verde, a variável com maior influência na intenção comportamental é a norma subjetiva.

Face ao exposto, conclui-se que a Teoria do Comportamento Planeado se revelou um importante preditor do comportamento de compra verde. Assim, dado que os valores médios dos itens relativos ao comportamento são inferiores aos valores médios da intenção comportamental, a TCP indicou que a atitude, a norma subjetiva e o controlo percebido podem ser trabalhadas tendo em vista uma mudança comportamental através do fornecimento de informação e de campanhas quer pelas empresas, quer de campanhas públicas de marketing social. Por exemplo, dado que se concluiu que a norma subjetiva é a variável com maior influência na intenção comportamental, poderá ser determinante para os governos encorajar o comportamento do público na compra verde (Gupta \& Ogden, 2009) direcionando programas de consciencialização da sociedade no que diz respeito aos seus hábitos de consumo. A importância dos grupos de referência e da norma subjetiva nas questões ambientais foi destacada em estudos anteriores (e.g., Santos, 2013). 0 comportamento de compra verde é um fator importante na medida em que pode encorajar, futuramente, o comportamento de compra verde de outros consumidores.

Uma das limitações deste estudo prende-se com o facto de o questionário ter sido efetuado a colaboradores de uma 
mesma e única empresa. Apesar de existir heterogeneidade demográfica e socioeconómica nos colaboradores, a amostra escolhida possui algumas semelhanças que no que se refere ao local de trabalho, que podem ter enviesado os resultados, demonstrando alguns comportamentos semelhantes. Em estudos futuros poderá ser interessante incluir outro tipo de variáveis na explicação da intenção de comportamento e do comportamento, tais como variáveis económicas ou situacionais.

\section{Referências}

Ajzen, I. (1985). From intentions to actions: a theory of planned behavior. In Julius Kuhl \& Jürgen Beckmann (Eds.). Action-control: From cognition to behavior, Part I, (pp. 11-39). Heidelberg, Germany: Springer.

Ajzen, I. \& Fishbein, M. (1980). Understanding attitudes and predicting social behaviour. Englewood Cliffs, NJ: Prentice-Hall.

Ajzen, I. (1991). The theory of planned behavior. Organizational Behavior and Human Decision Processes, 50(2), 179-211.

Akehurst, G., Afonso, C., \& Gonçalves, H. M. (2012). Re-examining green purchase behaviour and the green consumer profile: new evidences. Management Decision, 50(5), 972-988.

Bamberg, S., \& Schmidt, P. (1998). Changing travel-mode choice as rational choice: results from a longitudinal intervention study. Rationality and Society, 10(2), 223-252.

Biswas, A., Licata, J. W., McKee, D., Pullig, C., \& Daughtridge, C. (2000). The recycling cycle: An empirical examination of consumer waste recycling and recycling shopping behaviors. Journal of Public Policy \& Marketing, 19(1), 93-105.

Botetzagias, I., Dima, A.-F., \& Malesios, C. (2015). Extending the theory of planned behavior in the context of recycling: The role of moral norms and of demographic predictors. Resources, Conservation and Recycling, 95(1), 58-67.

Carroll, A. B. (1991). The pyramid of corporate social responsibility: Toward the moral management of organizational stakeholders. Business Horizons, 34(4), 39-48.

Chan, R. Y. (2001). Determinants of chinese consumers' green purchase behavior. Psychology \& Marketing, 18(4), 389-413.

Chan, R. Y., \& Lau, L. B. (2002). Explaining green purchasing behavior: A cross-cultural study on american and chinese consumers. Journal of International Consumer Marketing, 14(2-3), 9-40.

Chen, M.F., \& Tung, P.J. (2014). Developing an extended theory of planned behavior model to predict consumers' intention to visit green hotels. International Journal of Hospitality Management, 36(1), 221-230.

Cheung, S. F., Chan, D. K. S., \& Wong, Z. S. Y. (1999). Reexamining the theory of planned behavior in understanding wastepaper recycling. Environment and Behavior, 31(5), 587-612.

Chu, P. Y., \& Chiu, J. F. (2003). Factors influencing household waste recycling behavior: Test of an integrated model. Journal of Applied Social Psychology, 33(3), 604-626.

Conner, M., \& Armitage, C. J. (1998). Extending the theory of planned behavior: A review and avenues for further research. Journal of Applied Social Psychology, 28(15), 1429-1464.

Davis, G., O'Callaghan, F., \& Knox, K. (2009). Sustainable attitudes and behaviours amongst a sample of non-academic staff: A case study from an information services department, Griffith University, Brisbane. International Journal of Sustainability in Higher Education, 10(2), 136-151.

Fishbein, M., \& Ajzen, I. (1975). Belief, attitude, intention, and behavior: An introduction to theory and research. Reading, MA: Addison-Wesley.

Gonçalves, M. F., Menezes, J., \& Marques, C. (2015). Grocery consumer relational perceptions in green consumption context. Tourism \& Management Studies, 11(1), 160-163.

Gupta, S., \& Ogden, D. T. (2009). To buy or not to buy? A social dilemma perspective on green buying. Journal of Consumer Marketing, 26(6), 376-391.
Hair, J. F., Tatham, R. L., Anderson, R. E., \& Black, W. (2009). Multivariate data analysis: A global perspective. 7th ed. Upper Saddle River: Prentice Hall.

Han, Y., \& Hansen, H. (2012). Determinants of sustainable food consumption: A meta-analysis using a traditional and a structural equation modeling approach. International Journal of Psychological Studies, 4(1), 22-45.

Han, H., \& Kim, Y. (2010). An investigation of green hotel customers' decision formation: Developing an extended model of the theory of planned behavior. International Journal of Hospitality Management, 29(4), 659-668.

Han, H., Hsu, L. T. J., \& Sheu, C. (2010). Application of the theory of planned behavior to green hotel choice: Testing the effect of environmental friendly activities. Tourism Management, 31(3), 325334.

Heath, Y., \& Gifford, R. (2002). Extending the theory of planned behavior: predicting the use of public transportation. Journal of Applied Social Psychology, 32(10), 2154-2189.

Kaiser, F. G., \& Gutscher, H. (2003). The proposition of a general versionz of the theory of planned behavior: Predicting ecological behavior. Journal of Applied Social Psychology, 33(3), 586-603.

Kalafatis, S. P., Pollard, M., East, R., \& Tsogas, M. H. (1999). Green marketing and Ajzen's theory of planned behaviour: A cross-market examination. Journal of Consumer Marketing, 16(5), 441-460.

Kim, Y., \& Han, H. (2010). Intention to pay conventional-hotel prices at a green hotel-a modification of the theory of planned behavior. Journal of Sustainable Tourism, 18(8), 997-1014.

Litvine, D., \& Wüstenhagen, R. (2011). Helping "light green" consumers walk the talk: results of a behavioural intervention survey in the swiss electricity market. Ecological Economics, 70(3), 462-474

Marques, C. P. \& Almeida, D. (2013). A path model of attitudinal antecedents of green purchase behaviour, Economics \& Sociology, 6(2), 135-144.

Malhotra, N. K. (2010). Marketing research: An applied orientation. Upper Saddle River, NJ: Pearson.

Miras-Rodrígues, M. M., Escobar-Pérez, B., Gallego, A. M. (2015). Are companies less environmentally-friendly due to the crises? Evidence from Europe. Tourism \& Management Studies, 11(1), 2015.

Moser, A. K. (2015). Thinking green, buying green? Drivers of proenvironmental purchasing behavior. Journal of Consumer Marketing, 32(3), 167-175.

Oreg, S., \& Katz-Gerro, T. (2006). Predicting proenvironmental behavior cross-nationally values, the theory of planned behavior and value-belief-norm theory. Environment and Behavior, 38(4), $462-483$

Paço, A., \& Raposo, M. (2008). Determining the characteristics to profile the "green" consumer: An exploratory approach. International Review on Public and Nonprofit Marketing, 5(2), 129-140.

Paço, A., \& Raposo, M. (2009). “Green” segmentation: An application to the portuguese consumer market. Marketing Intelligence \& Planning, 27(3), 364-379.

Pujari, D., \& Wright, G. (1996). Developing environmentally conscious product strategies: A qualitative study of selected companies in Germany and Britain. Marketing Intelligence \& Planning, 14(1), 19-28.

Santos, M. A. 0. (2013). Investigating the influence of reference group judgments of global warming on consumers. Tourism and Management Studies, 9(1), 80-84.

Sparks, P., \& Shepherd, R. (1992). Self-identity and the theory of planned behavior: Assesing the role of identification with "green consumerism". Social Psychology Quarterly, 55(4), 388-399.

Wahid, N. A., Rahbar, E., \& Shyan, T. S. (2011). Factors influencing the green purchase behavior of Penang environmental volunteers. International Business Management, 5(1), 38-49.

Submetido: 16.05 .2014

Aceite: 24.11 .2014 